\title{
Metodología para la elaboración de canales endémicos y tendencia de la notificación del dengue, Valle del Cauca, Colombia, 2009-2013
}

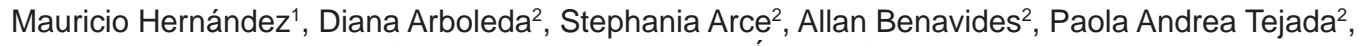 \\ Sindy Vanessa Ramírez ${ }^{2}$, Ángela Cubides ${ }^{1}$ \\ 1 Grupo de Investigación en Salud Pública, Universidad Santiago de Cali, Cali, Colombia \\ 2 Semillero de Investigación, Grupo de Investigación en Salud Pública, Fundación Universitaria San Martín, \\ Cali, Colombia
}

Introducción. El dengue es la enfermedad de más rápida propagación en el mundo y una permanente amenaza para la salud pública mundial, con aproximadamente 2,5 millones de personas en alto riesgo de infección. Ante la gravedad del cuadro de la enfermedad a nivel nacional y mundial, es necesario generar nuevas metodologías de predicción útiles para la adopción de decisiones en salud pública. Objetivo. Caracterizar los casos notificados de dengue entre el 2009 y el 2013 en el departamento del Valle del Cauca y presentar la metodología para elaborar canales endémicos en el caso del dengue.

Materiales y métodos. Se hizo un estudio descriptivo retrospectivo, utilizando la base de datos secundaria de las fichas de notificación, y se caracterizaron los casos de dengue entre el 2009 y el 2013. Se elaboraron dos canales endémicos, uno mediante promedios móviles y el otro con suavización exponencial.

Resultados. Se evidenció que la tendencia del dengue en el departamento del Valle del Cauca es positiva, lo que indica que en los últimos cinco años se ha incrementado el número de casos, aunque se observa una variación importante que podría explicarse por el ciclo de tres años que se inicia a partir del primer periodo epidemiológico del año.

Conclusión. La elaboración del canal endémico del dengue en el Valle del Cauca evidenció la importancia de aplicar estas metodologías de vigilancia en las situaciones de interés en salud pública. Como se observó en los resultados, hubo años en los que el número de casos fue muy bajo y otros en los que la epidemia alcanzó cifras muy elevadas.

Palabras clave: Aedes, virus del dengue, dengue/epidemiología, salud pública, enfermedades endémicas, notificación, Colombia.

doi: http://dx.doi.org/10.7705/biomedica.v36i0.2934

Methodology to develop endemic channels and notification trends for dengue in Valle del Cauca,
Colombia, 2009-2013

Introduction: Dengue is the fastest spreading disease in the world and a permanent threat to global public health. It is a viral illness for which approximately 2.5 million people are at high risk of infection. Given the severity of the disease at national and global levels, new predictive methodologies need to be generated to facilitate decision-making in public health.

Objective: To characterize cases of dengue reported from 2009 to 2013 in Valle del Cauca department, Colombia, and to establish a methodology to develop endemic channels that can be applied to this event. Materials and methods: This was a retrospective descriptive study. Notification forms were used as a secondary database to characterize dengue cases from 2009 to 2013 . Two endemic channels were developed, one using running means and the other through exponential smoothing.

Results: Dengue in the department of Valle del Cauca showed a positive tendency, indicating that the number of cases had increased in the last five years. An important variation was observed that could be explained by a three-year cycle beginning in the first epidemiological period of the year.

\footnotetext{
Contribución de los autores:

Mauricio Hernández: diseño, gestión y procesamiento estadístico de datos Diana Arboleda: redacción del manuscrito y consolidación de referencias

Allan Steven Benavides: obtención de la base de datos, tabulación y análisis

Stephania Arce y Sindy Vanesa Ramírez: análisis e interpretación de resultados

Paola Andrea Tejada: escritura de la discusión

Ángela Cubides: revisión crítica del artículo y participación en el análisis

Todos los autores participaron en la escritura del manuscrito.
} 
Conclusion: The development of the dengue endemic channel for Valle del Cauca illustrates the importance of applying these monitoring methodologies to events of public health interest. As can be seen from the results, there were some years in which the number of cases was very low and others in which the epidemic reached very high levels.

Key words: Aedes, dengue virus, dengue/epidemiology, public health, endemic diseases, notice, Colombia. doi: http://dx.doi.org/10.7705/biomedica.v36i0.2934

El dengue es una enfermedad viral transmitida principalmente por el vector Aedes aegypti (1), que puede evolucionar en forma asintomática o producir síntomas parecidos a los de un proceso febril. Es la principal arbovirosis en términos de morbilidad y mortalidad (2) y se han registrado cuatro serotipos del virus (DENV-1, DENV-2, DENV-3, DENV-4) $(3,4)$. Es la enfermedad de más rápida propagación en el mundo y es una permanente amenaza para la salud pública mundial, con aproximadamente 2,5 millones de personas en alto riesgo de infección (5). El dengue se presenta en climas tropicales y subtropicales de todo el mundo, tanto en zonas semiurbanas como urbanas, y su vector ocupa el mismo hábitat del hombre, ya que es diurno, fundamentalmente antropofílico (6) y su lugar preferido de cría es el agua limpia, casi siempre la almacenada para uso doméstico (7).

Desde hace varios años se reconoce que para lograr el control del dengue, no son suficientes los esfuerzos de las autoridades de salud, sino que es necesario que las comunidades afectadas se apropien de las medidas de control y prevención (8). Por esta razón, la estrategia de movilización y comunicación social para producir cambios de conducta (Planning Communication for Behavioural Impact, COMBI) es una de las más promovidas por la Organización Mundial de la Salud (OMS) y la Organización Panamericana de la Salud (OPS) (9). Sin embargo, la implementación de estrategias como esta constituye un desafío que enfrentan actualmente los programas de control de dengue en el país, el cual se ve amenazado por los limitados recursos humanos y económicos con que cuentan y la falta de integración multisectorial, la cual es la base de la estrategia $(10,11)$.

En los últimos 50 años, la incidencia del dengue ha aumentado 30 veces y se estima que anualmente se presentan 40 millones de infecciones, con unas

\footnotetext{
Correspondencia:

Mauricio Hernández, Carrera 79B № 9-18, apartamento C303, Cali, Colombia

Teléfono: (300) 2733699

mauriciohc@gmail.com

Recibido: 08/07/15; aceptado: 03/12/15
}

500.000 hospitalizaciones por dengue hemorrágico o choque por dengue, y que $90 \%$ de los casos se presenta en menores de 15 años $(5,12)$. Según el reporte del Ministerio de Salud titulado "La situación actual del dengue", en el que se analiza el periodo de 2008 a 2013, en la semana 12 del 2013 se detectaron situaciones de epidemia en 32 municipios del país, 18 de los cuales eran capitales de departamento $(13,14)$. El Valle del Cauca es el departamento con más casos de dengue en el país $(19,8 \%)(2,15)$; en Cali, la prevalencia de la enfermedad ha aumentado hasta ubicarlo como el municipio con mayor número de casos en Colombia $(15,5 \%$ del total nacional) $(16,17)$, lo que se explica, en parte, porque tiene las condiciones necesarias para que el vector se reproduzca adecuadamente.

Las principales condiciones para el control del vector incluyen la voluntad política, el mejoramiento de la infraestructura de salud y de los programas de control del vector, la coordinación intersectorial y una activa participación comunitaria $(18,19)$. Es necesario que la comunidad reconozca su responsabilidad para controlar el vector y que se produzca un cambio de conducta. Se deben fortalecer los planes para combatir los brotes, y los sistemas de vigilancia de las secretarías departamental y municipal de salud, las cuales envían al Sistema de Vigilancia en Salud Pública (Sivigila) las fichas de notificación, pero no llevan a cabo una búsqueda activa de casos (20-22).

Ante la gravedad de la situación de la enfermedad a nivel nacional y mundial, es necesario promover metodologías útiles de seguimiento para la adopción de decisiones en salud pública (7). Por ello, en el presente trabajo se presenta la forma de elaborar canales endémicos mediante dos metodologías reconocidas: la de promedios móviles y la de suavización exponencial, de tal manera que los profesionales encargados en el ámbito de la salud pública puedan implementarlas (23). La herramienta permitirá hacer la vigilancia a partir de límites permitidos de los casos de dengue notificados en el Valle del Cauca desde el 2009 hasta el 2013, incluida la información de Cali, municipio reconocido como el de mayor 
prevalencia de dengue en el país $(9,10)$, con el objetivo de hacer una adecuada planeación e implementar estrategias para el control y la disminución de casos.

\section{Materiales y métodos}

Se hizo un estudio descriptivo y retrospectivo, utilizando una base de datos secundaria de los casos de dengue notificados a la Secretaría Departamental de Salud del Valle del Cauca entre el 2009 y el 2013. Se caracterizaron las principales variables contenidas en la ficha de notificación y se elaboraron dos canales endémicos con diferentes metodologías para describir el comportamiento del dengue en el Valle del Cauca, con lo cual se determinó la línea de tendencia de la enfermedad durante el periodo mencionado.

\section{Plan de análisis}

Se describieron las características sociodemográficas de la población afectada por el dengue, teniendo en cuenta las variables contenidas en la ficha de notificación de los casos. Para las características cuantitativas se calculó el promedio y la desviación estándar y para las cualitativas se usaron tablas de frecuencias. El procesamiento de datos y la elaboración de figuras se hicieron en Excel 2010 y el programa R, versión 3.2.1. A continuación se describe paso a paso la elaboración de los canales endémicos.

\section{Elaboración del canal endémico}

En la elaboración del canal endémico se utilizó como dato de entrada el número de casos de dengue notificados por semana epidemiológica. El canal endémico es la representación gráfica del número de casos que se presentan en un área en períodos definidos (semana o periodo epidemiológico), comparado con los datos de años anteriores (usualmente, de cinco años).

Existen diferentes métodos para suavizar la tendencia observada y establecer límites de control: el método de promedios móviles, el de suavización exponencial, y métodos más sofisticados como los modelos autorregresivos y la metodología de Box-Jenkins.

En el presente estudio se elaboraron los canales endémicos del dengue mediante las técnicas de promedio móvil y de suavización exponencial, y se definieron los límites de control que permitieran evidenciar el riesgo de epidemia, los cuales se representaron gráficamente así: la zona de éxito, por debajo de la curva inferior; la zona de seguridad, entre la curva inferior y la media; la zona de alerta, entre la curva media y la superior, y la zona de epidemia, por encima de la curva superior.

Un valor ubicado en la zona de éxito indica que el número de casos de dengue notificados para esa semana presenta una frecuencia menor de la esperada. Uno ubicado en la zona de seguridad indica que el número de casos de dengue notificados para esa semana presenta un comportamiento estable. Un valor ubicado en la zona de alerta indica que el número de casos de dengue notificados para esa semana presenta una frecuencia que está por encima de lo esperado y es necesario estudiar la situación para determinar si corresponde a un comportamiento aleatorio o está comenzando un período epidémico. Un valor ubicado en la zona de epidemia indica que el número de casos de dengue notificados para esa semana presenta una situación que requiere acciones inmediatas de control.

El proceso continúa con la elaboración de un cuadro en el que la semana epidemiológica se pone en las filas y, los años, en las columnas. Esto se hace con base en la frecuencia de un evento (dengue, en este caso) en los cinco años anteriores, por lo que debe disponerse de los casos por semana epidemiológica para cada año.

A continuación, se agrega una columna en la cual se calcula el promedio aritmético de las cinco cifras de cada fila (por semana epidemiológica), para así obtener el promedio de casos por período. El factor de corrección se obtiene dividiendo la población estimada para el año en el que se aplica el índice por la población promedio de los cinco años anteriores. La columna siguiente es el valor esperado de casos por período (Y), el cual se obtiene multiplicando el promedio de casos de cada período por el factor de corrección. Cuando se grafica este valor esperado se obtiene una línea irregular, por lo tanto, deben calcularse los promedios móviles o datos suavizados exponencialmente para cada período. La columna siguiente es el promedio móvil (Y1) o el dato suavizado exponencialmente (S1), según sea el caso. El promedio móvil (Y1) se obtiene con el promedio aritmético de los períodos 1, 2, 3, 51 y 52 para el primer dato; de los períodos 1, 2, 3, 4 y 52, para el segundo dato; de los períodos 1, 2, 3, 4, y 5, para el tercer dato, y así sucesivamente; el último dato se obtiene con el promedio de los valores de los períodos 1, 2, 50, 51 y 52. Debe recordarse que el período corresponde a una semana epidemiológica (cuadro 1, figura 1). 
Cuadro 1. Cálculo del canal endémico

\begin{tabular}{|c|c|c|c|c|c|c|c|c|c|c|c|}
\hline \multirow{3}{*}{ Semana } & \multicolumn{6}{|c|}{ Casos observados en el quinquenio } & \multicolumn{3}{|c|}{ Casos esperados } & \multirow{2}{*}{\multicolumn{2}{|c|}{$\begin{array}{c}\text { Límites permisibles } \\
\text { Cálculo del error Cálculo de límites }\end{array}$}} \\
\hline & \multirow{2}{*}{$\begin{array}{c}\text { Año } \\
1\end{array}$} & \multirow{2}{*}{$\begin{array}{c}\text { Año } \\
2\end{array}$} & \multirow{2}{*}{$\begin{array}{c}\text { Año } \\
3\end{array}$} & \multirow{2}{*}{$\begin{array}{c}\text { Año } \\
4\end{array}$} & \multirow{2}{*}{$\begin{array}{c}\text { Año } \\
5\end{array}$} & \multirow{2}{*}{ Promedio } & \multirow{2}{*}{$\begin{array}{l}\text { Factor de } \\
\text { corrección }\end{array}$} & \multirow{2}{*}{$\begin{array}{c}\text { Promedio } \\
\text { esperado } \\
\text { Y }\end{array}$} & \multirow{2}{*}{$\begin{array}{l}\text { Promedio } \\
\text { suavizado } \\
\mathrm{Y}_{1}-\mathrm{S}_{1}\end{array}$} & & \\
\hline & & & & & & & & & & $\left(Y-Y_{1}\right) \quad\left(Y-Y_{1}\right)^{2}$ & $\left(Y_{1}-2 S\right)\left(Y_{1}+2 S\right)$ \\
\hline \multicolumn{12}{|l|}{1} \\
\hline \multicolumn{12}{|l|}{2} \\
\hline \multicolumn{12}{|l|}{. } \\
\hline \multicolumn{12}{|l|}{. } \\
\hline \multicolumn{12}{|l|}{. } \\
\hline \multicolumn{12}{|l|}{51} \\
\hline \multicolumn{12}{|l|}{52} \\
\hline Total & & & & & & & & & & Suma & \\
\hline
\end{tabular}

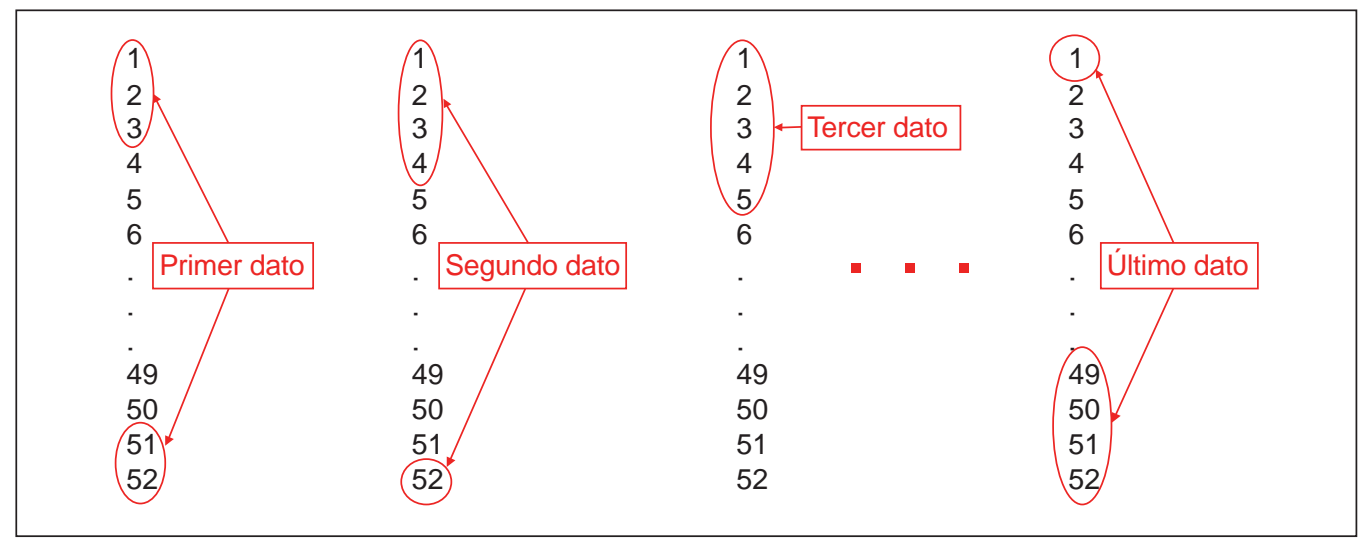

Figura 1. Cálculo de promedios móviles para elaborar el canal endémico

El dato suavizado exponencialmente (S1) se obtiene de la siguiente manera: primero, se establece una constante de suavización, la cual depende del grado de suavización que se desee; entre menor sea, menos alteraciones se generan. Lo contrario sucede si está próximo a 1, ya que no se encontrarán grandes diferencias con respecto a la curva de valores esperados $(Y)$.

Una vez establecida dicha constante, se procede a hallar los datos suavizados de la siguiente forma:

$S_{1}=Y(1)$, valor esperado del primer período

$\mathrm{S}_{2}=()[\mathrm{Y}(2)]+(1-)\left(\mathrm{S}_{1}\right)$

$\mathrm{S}_{3}=()[\mathrm{Y}(3)]+(1-)\left(\mathrm{S}_{2}\right)$

$\mathrm{S}_{\mathrm{t}}=()[\mathrm{Y}(\mathrm{t})]+(1-)\left(\mathrm{S}_{\mathrm{t}-1}\right)$

Los valores de los promedios móviles o datos suavizados exponencialmente se colocan en un plano cartesiano de dos ejes $(X, Y)$ y dan origen al canal endémico del dengue.
A la curva endémica obtenida se le fijan límites permisibles con base en el error estándar de las estimaciones y la siguiente fórmula:

$$
S=\sqrt{\frac{\operatorname{sum}\left(Y-Y_{1}\right)^{2}}{n}} \text { o } S=\sqrt{\frac{\operatorname{sum}\left(Y-S_{1}\right)^{2}}{n}}
$$

Los límites superior e inferior del canal endémico se calculan en dos columnas adicionales de la siguiente manera.

Límite inferior igual a (Y1-2s) o (S1-2s)

Límite superior igual a $(\mathrm{Y} 1+2 \mathrm{~s})$ o $(\mathrm{S} 1+2 \mathrm{~s})$

Para seleccionar la constante de suavización exponencial fue necesario hacer varias pruebas, de modo que la constante seleccionada no perturbara mucho la información original.

Mientras que el método de promedios móviles se ve afectado por datos extremos, el método de suavización exponencial es adecuado en aquellos casos en los que el comportamiento de 
los datos no tiene una tendencia ascendente o descendente. Este método se basa en suavizar los valores de una serie de manera exponencial decreciente, aplicando una constante $\alpha(0<\alpha$ $<1$ ); la observación más reciente recibe el peso $\alpha$, la siguiente $\alpha(1-\alpha)$; luego $\alpha(1-\alpha)^{2}$, y así sucesivamente. Los resultados dependen del valor de $\alpha$, y se recomienda que este valor se encuentre entre 0,01 y 0,30 , ya que si es superior a 0,30 se generan cambios sensibles en las fluctuaciones $y$, si es muy pequeño, se genera el efecto contrario y las curvas generadas no responderán adecuadamente a los cambios en los datos. Se pueden utilizar valores distintos a $\alpha$ dependiendo de la variación de los valores históricos empleados. Por ejemplo, si los datos son muy estables, se podría utilizar un valor de $\alpha$ bajo. Estos valores dependerán de la experiencia del analista y de su conocimiento sobre el comportamiento de los datos.

En este caso, para elegir la constante de suavización exponencial fue necesario hacer varias pruebas, de modo que la constante seleccionada no perturbara la información original, para lo cual se utilizaron diferentes constantes de suavización entre 0,01 y 0,30, y se escogió la constante $\alpha=0,28$.

\section{Consideraciones éticas}

La investigación se rigió por lo establecido en la Resolución 8430 de 1993, del Ministerio de Salud de Colombia, según la cual se trata de una investigación sin riesgo en la cual se usó una base de datos secundaria. Se contó con el aval del Comité de Ética de la Fundación Universitaria San Martín (Cali) y con autorización de la Secretaría Departamental de Salud del Valle.

\section{Resultados}

Durante el periodo comprendido entre el 2009 y el 2013, hubo mayor incidencia de casos de infección por el virus del dengue en los hombres que en las mujeres; los años de mayor incidencia fueron el 2010 y el 2013 y, en este último, se presentó la mayoría de los casos reportados en el periodo de estudio; en el 2011 y el 2012, por el contrario, hubo una disminución drástica en el número de casos (cuadro 2 y figura 2).

Se observó un predominio de casos en los hombres, con $53,23 \%$, mientras que en las mujeres fue de $46,77 \%$. La mayoría de los pacientes reportados mediante ficha de notificación no fueron hospitalizados. La condición final reveló que tres pacientes murieron a causa de la enfermedad en el 2009, el 2010 y el 2012.

La mayoría de los casos se presentó en la cabecera municipal $(91,23 \%)$, seguida de los centros poblados $(6,06 \%)$ y de las áreas rurales dispersas $(2,71 \%)$. La mayoría de los pacientes $(93,54 \%)$ afectados por el virus del dengue no se había desplazado en los cinco días anteriores al inicio de los síntomas y en el momento de aparición de la infección la mayoría de ellos no convivía con personas con síntomas indicativos de la enfermedad (48,41\%) (cuadro 3 ).

En cuanto a la edad, se encontró que el mayor número de pacientes tenía entre 10 y 14 años, cifra que descendió al aumentar la edad. Sin embargo, se evidenció un incremento de casos en menores de cinco años en el 2011 (figura 3).

Entre los municipios de residencia señalados en las fichas de notificación, Palmira (6,91\%), Cartago $(4,74 \%)$, Buga (3,33\%), Tuluá (2,90\%), Yumbo $(2,56 \%)$ y Florida $(1,70 \%)$ presentaron una alta tasa de casos de infección por $A$. aegypti; sin embargo, en el municipio de Cali $(63,39 \%)$ se registró la mayoría de casos en el periodo de estudio. Los municipios de Alcalá $(0,15 \%)$, El Águila $(0,05 \%)$, El Cairo $(0,03 \%)$, El Dovio (0,01\%), La Cumbre $(0,02 \%)$, Obando $(0,13 \%)$, Restrepo $(0,07 \%)$, Ulloa $(0,03 \%)$ y Versalles $(0,04 \%)$ presentaron el menor número de casos de dengue notificados en

Cuadro 2. Distribución de la edad según sexo y año de aparición de los casos de dengue, Valle del Cauca, 2009 a 2013

\begin{tabular}{cccccccccc}
\hline \multirow{2}{*}{$\begin{array}{c}\text { Año de } \\
\text { aparición }\end{array}$} & \multicolumn{3}{c}{ Sexo femenino } & \multicolumn{3}{c}{ Sexo masculino } & \multicolumn{3}{c}{ Total } \\
\cline { 2 - 10 } & $\mathbf{n}$ & Promedio & DE & $\mathbf{n}$ & Promedio & DE & $\mathbf{n}$ & Promedio & DE \\
\hline 2009 & 5.103 & 22,8 & 18,7 & 5.583 & 22,1 & 17,6 & 10.686 & 22,4 & 18,1 \\
2010 & 9.760 & 24,8 & 20,2 & 10.461 & 23,8 & 18,7 & 20.221 & 24,3 & 19,5 \\
2011 & 1.456 & 29 & 22,8 & 2.020 & 27,5 & 19,9 & 3.476 & 28,2 & 21,2 \\
2012 & 1.737 & 26,8 & 21,7 & 2.376 & 24,8 & 18,7 & 4.113 & 25,7 & 20,1 \\
2013 & 11.715 & 27,1 & 19,8 & 13.444 & 25,7 & 18,7 & 25.159 & 26,3 & 19,2 \\
Total & 29.771 & 25,7 & 20,1 & 33.884 & 24,5 & 18,7 & 63.655 & 25,1 & 19,4 \\
\hline
\end{tabular}

DE: desviación estándar 
el periodo de estudio $y$, aunque el menor número de casos se presentó en el municipio de El Dovio $(0,01 \%)$, no se descarta que haya sesgos en el estudio debido a deficiencias en la oportunidad y la exactitud de la notificación, además del hecho de que algunos habitantes afectados con el virus podrían no haber tenido acceso a servicios de salud, lo cual afectaría las estadísticas (cuadros 4 y 5 ).

Los canales endémicos elaborados por semana epidemiológica mostraron que el comportamiento del dengue tuvo una tendencia ascendente en las primeras semanas epidemiológicas, hasta llegar a un pico en la semana 8 , cuando se registró una media alrededor de los 300 casos, en tanto que al llegar a la semana 29 se presentó una disminución (figura 4).

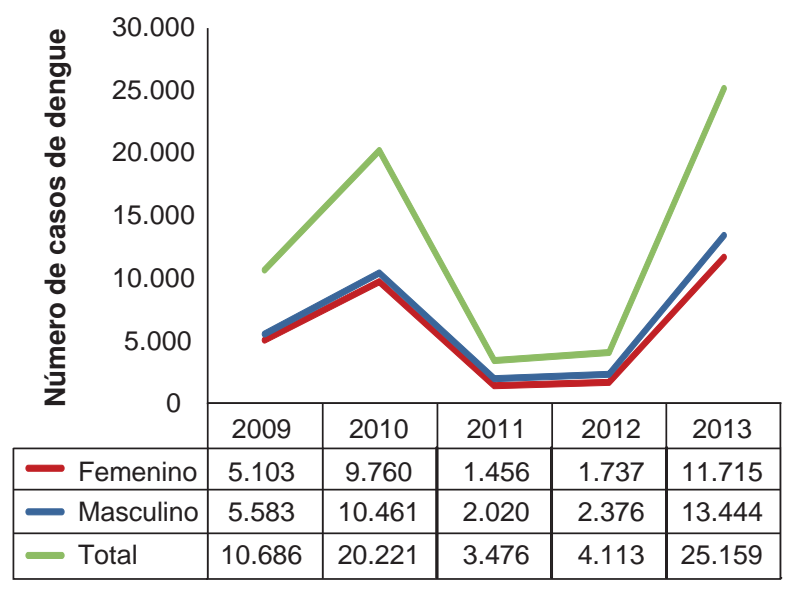

Figura 2. Distribución del número de casos de dengue según sexo y año de aparición, Valle del Cauca, 2009 a 2013

\section{Discusión}

El comportamiento del dengue durante los años estudiados tuvo una tendencia positiva, con un incremento en el número de casos en 2013. Sin embargo, en el 2010 y el 2011 el número de casos fue muy bajo con respecto a los demás años, lo cual indica que la capacidad de transmisión estaría relacionada con un ciclo de tres años, patrón que habría que investigar en futuros estudios sobre el dengue. Los factores de riesgo de la infección no se establecieron por sexo, pues la probabilidad de enfermar en hombres y mujeres es igual, aunque a la luz de los resultados del presente estudio hubo una mayor tendencia de infección en hombres que en mujeres; se podría concluir, entonces, que el sexo masculino es más propenso al contagio, seguramente porque los hombres tienen mayor probabilidad de contacto con el vector debido a que son quienes usualmente trabajan y deben desplazarse más que las mujeres.

En el grupo entre los 10 y los 14 años de edad se presentó el mayor número de casos; sin embargo, en el 2011 se registró un cambio evidente, pues los casos predominaron en los menores de cinco años; un estudio en Tailandia describe el virus del dengue como el principal entre niños de uno a 15 años de edad (24), lo cual concuerda con los resultados del presente estudio y evidencia la aparición de un número importante de casos de infección en edades pediátricas y en la adolescencia.

Las epidemias de dengue tienen una tendencia ascendente y afectan en mayor proporción a los menores de 15 años, lo cual daría pie a la

Cuadro 3. Distribución según tipo de variable de la ficha de notificación y año de aparición de los casos de dengue, Valle del Cauca, 2009 a 2013

\begin{tabular}{|c|c|c|c|c|c|c|c|}
\hline Variable & Categoría & $\begin{array}{c}2009 \\
n=10.686\end{array}$ & $\begin{array}{c}2010 \\
n=20.221\end{array}$ & $\begin{array}{c}2011 \\
n=3.476\end{array}$ & $\begin{array}{c}2012 \\
n=4.113\end{array}$ & $\begin{array}{c}2013 \\
n=25.159\end{array}$ & $\begin{array}{l}\text { Porcentaje } \\
\text { total }\end{array}$ \\
\hline \multirow[t]{2}{*}{ Sexo } & Femenino & 5.103 & 9.760 & 1.456 & 1.737 & 11.715 & 46,77 \\
\hline & Masculino & 5.583 & 10.461 & 2.020 & 2.376 & 13.444 & 53,23 \\
\hline \multirow[t]{2}{*}{ Paciente hospitalizado } & No & 7.419 & 15.138 & 2.631 & 2.879 & 19.289 & 74,39 \\
\hline & Sí & 3.267 & 5.083 & 845 & 1.234 & 5.870 & 25,61 \\
\hline \multirow[t]{2}{*}{ Condición final } & Muerto & 1 & 1 & & 1 & & 0,00 \\
\hline & Vivo & 10.685 & 20.220 & 3.476 & 4.112 & 25.159 & 100,00 \\
\hline \multirow[t]{3}{*}{ Área de aparición del caso } & Cabecera municipal & 9.745 & 17.609 & 3.110 & 3.708 & 23.899 & 91,23 \\
\hline & Centro poblado & 646 & 1.881 & 242 & 256 & 832 & 6,06 \\
\hline & Zona rural dispersa & 295 & 731 & 124 & 149 & 428 & 2,71 \\
\hline ¿Desplazamiento en los & No & 10.168 & 19.049 & 3.104 & 3.721 & 23.503 & 93,54 \\
\hline \multirow[t]{2}{*}{ últimos cinco días? } & Sí & 412 & 836 & 334 & 357 & 1.539 & 5,46 \\
\hline & Sin datos & 106 & 336 & 38 & 35 & 117 & 0,99 \\
\hline ¿Algún familiar o conviviente & No se sabe & 2 & 872 & 596 & 470 & 1.979 & 6,16 \\
\hline ha tenido síntomas de dengue & No & 2 & 3.249 & 2.677 & 3.373 & 21.512 & 48,41 \\
\hline \multirow[t]{2}{*}{ en los últimos 15 días? } & Sí & & 233 & 160 & 235 & 1.551 & 3,42 \\
\hline & Sin datos & 10.682 & 15.867 & 43 & 35 & 117 & 42,01 \\
\hline
\end{tabular}


hipótesis de que en estas edades los individuos están más en contacto con el medio ambiente y ello podría contribuir a una mayor posibilidad de infección, además de las condiciones precarias en que vive la mayoría de la población (25). Se ha reportado una estrecha relación entre el dengue y los cambios climáticos globales (en el 2010, la temperatura promedio en Cali fue de $24,6{ }^{\circ} \mathrm{C}$ y, en el 2013, de $25^{\circ} \mathrm{C}$ ); el incremento en los casos de dengue se ha asociado con el fenómeno de El Niño, el cual se caracteriza por grandes sequías y un aumento de la temperatura en todo el territorio

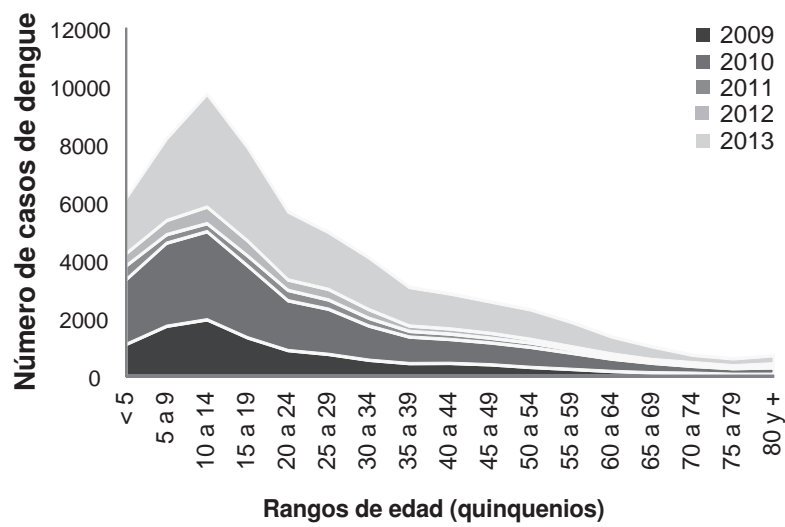

Figura 3. Distribución del número de casos de dengue según rangos de edad (quinquenios) y año de aparición, Valle del Cauca, 2009 a 2013
(26). En consecuencia, los habitantes recurren al almacenamiento de agua, casi siempre de forma inapropiada. De igual manera, en temperaturas más cálidas el tamaño de las larvas de $A$. aegypti se reduce $y$, por ende, el tamaño del adulto y, como se sabe, los adultos más pequeños tienen que alimentarse con más frecuencia para desarrollar un lote de huevos, lo que aumenta la frecuencia de picaduras y, con ello, la transmisión del virus (27).

La mayoría de los pacientes afectados por el virus del dengue no se desplazaron en los cinco días previos al inicio de los síntomas y, en el momento de aparición de la infección, la mayoría de ellos no convivía con personas que presentaran síntomas indicativos de la enfermedad. Esto nos lleva a concluir que el departamento del Valle del Cauca es una zona endémica para la infección por el virus del dengue, lo cual la convierte en una enfermedad de interés en salud pública de alto impacto, en cuya aparición influyen múltiples factores, entre ellos, las condiciones favorables para los criaderos del mosquito, la falta de concientización sobre la enfermedad entre los habitantes, la aparición de ciclos epidémicos cada vez más cortos, la circulación simultánea de los cuatro serotipos y, además, la infestación por $A$. aegypti del territorio situado por debajo de los 1.800 metros sobre el nivel del mar.

Cuadro 4. Distribución según departamento de residencia y año de aparición de los casos de dengue

\begin{tabular}{|c|c|c|c|c|c|c|c|}
\hline $\begin{array}{l}\text { Departamento } \\
\text { de residencia }\end{array}$ & $\begin{array}{c}2009 \\
n=10.686\end{array}$ & $\begin{array}{c}2010 \\
n=20.221\end{array}$ & $\begin{array}{c}2011 \\
n=3.476\end{array}$ & $\begin{array}{c}2012 \\
n=4.113\end{array}$ & $\begin{array}{c}2013 \\
n=25.159\end{array}$ & Total & $\begin{array}{l}\text { Porcentaje } \\
\text { total }\end{array}$ \\
\hline Valle & 10.549 & 19.873 & 3.462 & 4.092 & 25.081 & 63.057 & 99,06 \\
\hline Otros & 137 & 348 & 14 & 21 & 78 & 598 & 0,94 \\
\hline
\end{tabular}

Cuadro 5. Distribución según municipio de residencia en el Valle del Cauca y año de aparición de los casos de dengue, 2009 a 2013

\begin{tabular}{|c|c|c|c|c|c|c|c|c|}
\hline Municipio & $\begin{array}{c}2009 \\
n=10.686\end{array}$ & $\begin{array}{c}2010 \\
n=20.221\end{array}$ & $\begin{array}{c}2011 \\
n=3.476\end{array}$ & $\begin{array}{c}2012 \\
n=4.113\end{array}$ & $\begin{array}{c}2013 \\
n=25.159\end{array}$ & $\begin{array}{l}\text { Porcentaje } \\
\text { total }\end{array}$ & $\begin{array}{l}\text { Población } \\
\text { del Valle } \\
2013\end{array}$ & $\begin{array}{c}\text { Tasa x } 1.000 \\
\text { habitantes } \\
2013\end{array}$ \\
\hline Cali & 5.899 & 11.058 & 1.737 & 2.571 & 18.707 & 63,39 & 2'319.655 & 8 \\
\hline Palmira & 1.966 & 1.166 & 270 & 195 & 763 & 6,91 & 300.712 & 3 \\
\hline Cartago & 137 & 1.091 & 244 & 133 & 1.386 & 4,74 & 130.827 & 11 \\
\hline Buga & 237 & 1.037 & 180 & 177 & 471 & 3,33 & 115.613 & 4 \\
\hline Tuluá & 302 & 513 & 173 & 201 & 641 & 2,90 & 206.588 & 3 \\
\hline Yumbo & 233 & 563 & 83 & 144 & 590 & 2,56 & 111.707 & 5 \\
\hline Florida & 220 & 295 & 58 & 44 & 455 & 1,70 & 57.697 & 8 \\
\hline Candelaria & 141 & 341 & 38 & 36 & 325 & 1,40 & 79.279 & 4 \\
\hline La Unión & 154 & 291 & 169 & 87 & 98 & 1,27 & 36.444 & 3 \\
\hline Pradera & 270 & 193 & 24 & 48 & 164 & 1,11 & 53.792 & 3 \\
\hline Buenaventura & 60 & 308 & 76 & 82 & 149 & 1,07 & 384.402 & 0 \\
\hline Otros municipios $<1 \%$ & 930 & 3.017 & 410 & 374 & 1.332 & 9,62 & 723.450 & 2 \\
\hline Total & 10.549 & 19.873 & 3.462 & 4.092 & 25.081 & 100,00 & 4'520.166 & 6 \\
\hline
\end{tabular}



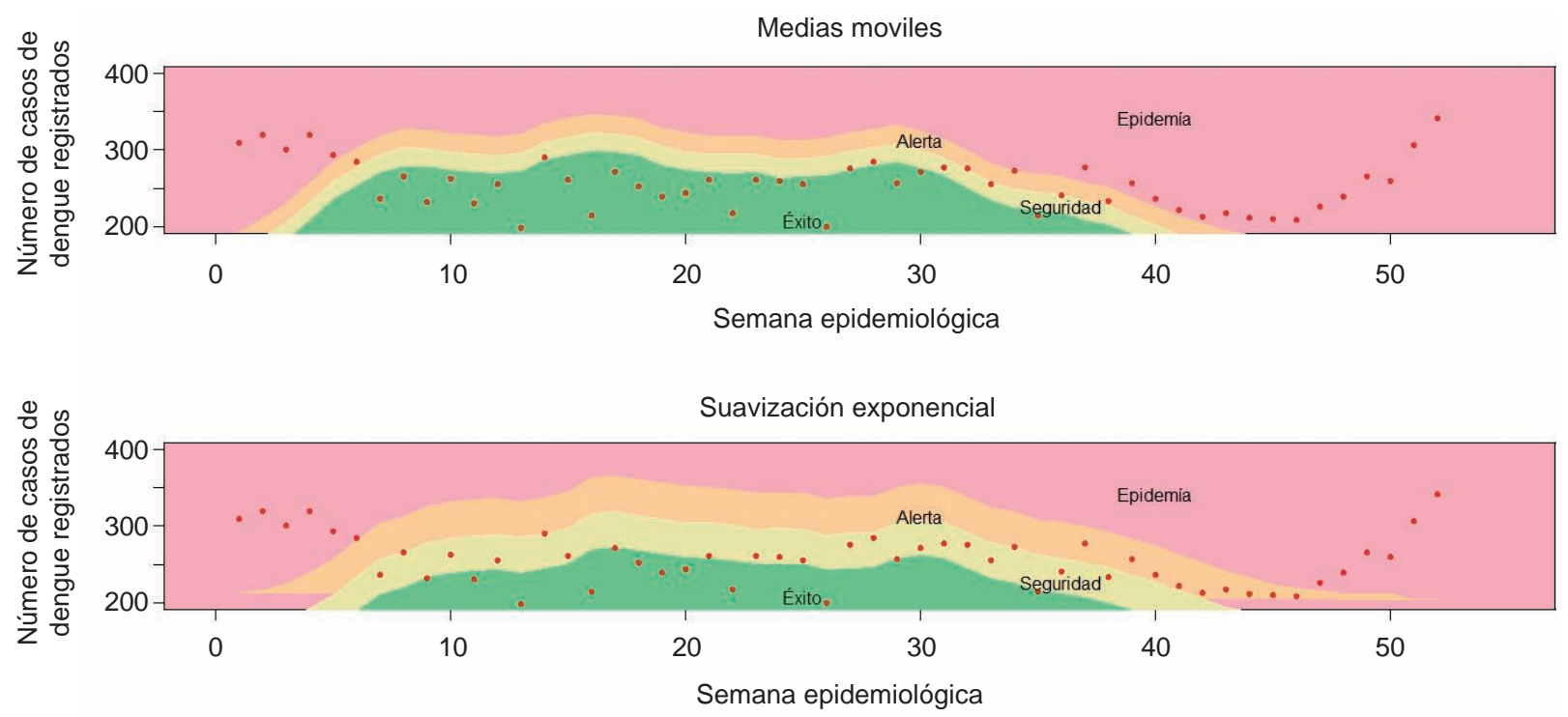

Figura 4. Canales endémicos del dengue por semana epidemiológica en el Valle del Cauca de 2009 a 2013, elaborados mediante los métodos de promedios móviles y de suavización exponencial

En un artículo sobre dengue y dengue hemorrágico en las Américas (20), se hace énfasis en lo establecido por la OMS para el control y la prevención del dengue, especialmente en los planes dirigidos a lograr la adecuada manipulación y limpieza de los contenedores domésticos de agua, y el aumento de la capacidad de reconocimiento de la enfermedad por parte del personal médico, de los mismos pacientes y de la comunidad. Según los resultados preliminares disponibles, estos planes han contribuido a fortalecer los programas nacionales (28). Sin embargo, los resultados de la curva epidemiológica del Valle del Cauca durante estos últimos años contradicen la orientación de esta metodología, lo cual exige indagar sobre nuevos métodos de control sanitario y de prevención de la propagación del dengue teniendo en cuenta las zonas tropicales de mayor incidencia $(26,29)$. Las intervenciones de prevención de la infección por el virus no deben ser solamente epidemiológicas, pues se necesita la ayuda de todos los sectores involucrados (30). El control del dengue es más que la utilización de insecticidas o campañas de limpieza, incluye la modificación de factores sociales y culturales que favorecen la transmisión. Estos factores predominan en el ámbito familiar, pues están en estrecha relación con las formas de vida de cada hogar. En este sentido se ha constatado que el principal factor determinante del crecimiento del vector $A$. aegypti es la manera inadecuada de manejar las aguas estancadas, así que, si se logra establecer hábitos adecuados en cada hogar, se podría disminuir la presencia de la enfermedad notoriamente en la cabecera municipal, así como la morbimortalidad que produce $(22,31)$.

Puede haber un subregistro importante de dengue, pues es posible que algunos casos no se hayan notificado porque los pacientes no acudieron a las instituciones prestadoras de servicios de salud, las cuales son las encargadas de notificar al Sivigila, o porque la condición no se configuró como caso.

Los canales endémicos se elaboraron con dos métodos, el de promedios móviles y el de suavización exponencial. El primero está influenciado por datos extremos y el segundo requiere el uso de diferentes valores de alfa hasta encontrar el adecuado. No se tuvieron en cuenta otros métodos, como el de la media geométrica o el de los cuartiles.

\section{Agradecimientos}

A la Secretaría de Salud Departamental del Valle, por autorizar el uso de la base de datos y promover investigaciones que permitan conocer la dinámica de enfermedades de gran impacto en salud pública como el dengue, y al área de investigación de la Facultad de Medicina de la Fundación Universitaria San Martín - Cali, por brindarnos los conocimientos necesarios para escribir este artículo.

\section{Conflicto de intereses}

Los autores declaran que no hay conflicto de intereses con respecto al presente manuscrito. 


\section{Financiación}

La investigación se hizo con recursos propios de los investigadores.

\section{Referencias}

1. Fonseca I, Quiñones ML, Lenhart A, Brogdon WG. Insecticide resistance status of Aedes aegypti (L.) from Colombia. Pest Manag Sci. 2011;67:430-7. http://dx.doi. org/10.1002/ps.2081

2. Rodríguez JM, Ortiz Y, Rodríguez RF. Epidemiología del dengue en Palmira, Valle, Colombia, 2001-2004. Revista de la Facultad de Medicina. 2014;54:88-95.

3. Velandia ML, Castellanos JE. Virus del dengue: estructura y ciclo viral. Infectio. 2011;15:33-43.

4. Ocazionez RE, Ortiz AS, Gómez SY, Miranda DR. Virus del dengue de serotipo 1 (DENV-1) de Colombia: su contribución a la presentación del dengue en el departamento de Santander. Biomédica. 2013;33:22-30. http://dx.doi.org/10.7705/biomedica.v33i0.717

5. Zambrano $\mathbf{P}$, Mercado $\mathbf{M}$. Vigilancia y análisis del riesgo en salud pública. Protocolo de vigilancia en salud pública del dengue 2011. Fecha de consulta: 22 de junio 2015. Disponible en: http://www.ins.gov.co/lineas-de-accion/ Subdireccion-Vigilancia/sivigila/Protocolos\%20SIVIGILA/ PRO\%20Dengue.pdf

6. Hernández PA. Aporte a la enseñanza de la epidemiología de las enfermedades transmisibles en Cuba, 2009. Fecha de consulta: 18 de junio 2015. Disponible en: http://tesis. repo.sld.cu/94/1/Pedro_Rodr\%C3\%ADguez.pdf

7. Rodríguez J, Prieto S, Correa C, Arnold Y, Álvarez L, Bernal $\mathbf{P}$, et al. Dinámica de la epidemia del dengue en Colombia: predicciones de la trayectoria de la epidemia. Revista Med. 2013;21:38-45.

8. Carabalí M, Ocampo CB, Toledo ME, Osorio L. Difusión masiva de reportes situacionales sobre dengue: efectos de la intervención en Guadalajara de Buga, Colombia. Biomédica. 2013;33:130-41. http://dx.doi.org/10.7705/ biomedica.v33i0.724

9. Parks W, Lloyd L. Planificación de la movilización y comunicación social para la prevención y el control del dengue 2004. Fecha de consulta: 26 de junio 2015. Disponible en: http://cdrwww.who.int/entity/tdr/publications/ documents/planificacion_dengue.pdf

10. Cáceres-Manrique FdeM, Vesga-Gómez C, Angulo-Silva ML. Empoderamiento para la prevención y control del dengue. Rev Salud Pública. 2010;12:798-806. http://dx.doi. org/10.1590/S0124-00642010000500010

11. González AL, Martínez RA, Villar LA. Evolución clínica de pacientes hospitalizados por dengue en una institución de salud de Bucaramanga, Colombia. Biomédica. 2008;28:53143. http://dx.doi.org/10.7705/biomedica.v28i4.58

12. González A, Ibarra AM. Nivel de conocimientos, actitudes y prácticas sobre la prevención del mosquito Aedes aegypti en comunidades del municipio Diez de Octubre, La Habana. Rev Cubana Hig Epidemiol. 2011;49:247-59.

13. Ministerio de Salud y Protección Social. Situación actual del dengue a semana 12 de 2013, periodo de análisis : 2008-2013. Fecha de consulta: 10 de mayo 2015.
Disponible en: http://www.minsalud.gov.co/Documentos\%20 y\%20Publicaciones/INFORME\%20SITUACION\% 20DE\% 20DENGUE.pdf

14. Restrepo BN, Piedrahita LD, Agudelo IY, Marín K, Ramírez R. Infección por dengue: una causa frecuente de síndrome febril en pacientes de Quibdó, Chocó, Colombia. Biomédica. 2014;35:131-7. http://dx.doi.org/10.7705/ biomedica.v35i1.2345

15. Ministerio de Salud y Protección Social. Dengue 2015. Fecha de consulta: 1 de junio 2015. Disponible en: http:// www.minsalud.gov.co/salud/publica/PET/Paginas/dengue. aspx

16. Wilder A, Ooi E, Vasudevan SG, Gubler DJ. Update on dengue: Epidemiology, virus evolution, antiviral drugs, and vaccine development. Curr Infect Dis Rep. 2010; 2:157-64. http://dx.doi.org/10.1007/s11908-010-0102-7

17. Rúa GL, Suárez CdelR, Rojo RA. Implicaciones epidemiológicas de Aedes albopictus (Skuse) en Colombia. Rev Fac Nac Salud Pública. 2012;30:328-37.

18. Peláez O, Sánchez L, Mas-Bermejo P, Pérez S, Kourí G, Guzmán MG. Prevalencia de síndromes febriles en la vigilancia del dengue: Ciudad de La Habana, 2007. Rev Cubana Hig Epidemiol. 2010;48:1-14

19. Caldera S, Jaramillo S, Cochero S, Pérez A, Bejarano E. Diferencias genéticas entre poblaciones de Aedes aegypti de municipios del norte de Colombia, con baja y alta incidencia de dengue. Biomédica. 2013;33:89-98. http://dx. doi.org/10.7705/biomedica.v33i0.1573

20. Periago MR, Guzmán MG. Dengue y dengue hemorrágico en las Américas. Rev Panam Salud Pública. 2007;21:18791. http://dx.doi.org/10.1590/S1020-49892007000300001

21. Clark GG, Rubio Y. Mosquito vector biology and control in Latin America - a 22nd Symposium. J Am Mosq Control Assoc. 2012;28:102-10. http://dx.doi.org/10.2987/12-6253.1

22. Ocampo CB, González C, Morales CA, Pérez M, Wesson D, Apperson CS. Evaluation of community-based strategies for Aedes aegypti control inside houses. Biomédica. 2009; 29:282-97. http://dx.doi.org/10.7705/biomedica.v29i2.30

23. Guzmán A, Istúriz RE. Update on the global spread of dengue. Int J Antimicrob Agents. 2010;36:S40-2. http://dx. doi.org/10.1016/j.ijantimicag.2010.06.018

24. Acosta HF, Bayona MA, Zabaleta TE, Villar LA, Narváez CF, Rodríguez JA, et al. Compromiso hepático por dengue en niños del Huila, Colombia. Rev Salud Pública. 2012;14:982-92

25. Instituto Nacional de Salud, Ministerio de la Protección Social, Organización Panamericana de la Salud. Protocolo para la vigilancia en salud pública del dengue. Fecha de consulta: 11 de abril 2015. Disponible en: http://www.ins. gov.co/temas-de-interes/Dengue/01\%20Protocolo\%20 Dengue.pdf

26. Rúa GL, Suárez C, Chauca J, Ventosilla P, Almanza R. Modelado del efecto de la variabilidad climática local sobre la transmisión de dengue en Medellín (Colombia) mediante análisis de series temporales. Biomédica. 2013;33:142-52. http://dx.doi.org/10.7705/biomedica.v33i0.1444

27. Instituto Nacional de Salud, Ministerio de la Protección Social, Organización Panamericana de la Salud. Gestión para la vigilancia entomológica y control de la transmisión 
de dengue 2015. Fecha de consulta: 20 de junio 2015 Disponible en: http://www.ins.gov.co/temas-de-interes/dengue/ 03\%20vigilancia\%20entomo\%20dengue.pdf

28. Guzmán MG. El dengue y el dengue hemorrágico ¿una entidad olvidada? Rev Cubana Med Trop. 2002;54:169-70.

29. Constanza L, Quintero J, García T, González C, Fuentes M. Funcionamiento de las políticas gubernamentales para la prevención y el control del dengue: el caso de Arauca y Armenia en Colombia. Biomédica. 2015;35:186-95. http:// dx.doi.org/10.7705/biomedica.v35i2.2332
30. Departamento Administrativo de Planeación de Santiago de Cali. Cali en cifras 2011. Fecha de consulta: 22 de junio 2015. Disponible en: http://planeacion.cali.gov.co/ Publicaciones/Cali_en_Cifras/Caliencifras2011.pdf

31. Moreira I, Gámez D. Características clínicas epidemiológicas de los cooperantes con dengue en el municipio Torres, estado de Lara, Venezuela, 2008. Rev Cubana Hig Epidemiol. 2012;50:179-88. 